\title{
A systematic review of the role of eculizumab in systemic lupus erythematosus-associated thrombotic microangiopathy
}

\author{
Rachael D. Wright ${ }^{1 *}$, Fariba Bannerman ${ }^{2}$, Michael W. Beresford ${ }^{1,3}$ and Louise Oni ${ }^{1,4}$
}

\begin{abstract}
Background: Lupus nephritis (LN) is a severe consequence of systemic lupus erythematosus (SLE) that affects approximately $40 \%$ of patients. Pathogenic immune complexes that are characteristic of LN deposit in the kidney and activate immune mediated pathways including the complement system. Complete remission rates in LN are approximately $44 \%$ highlighting the need for new treatment strategies in these patients. Eculizumab is a fully humanised lgG2/lgG4 monoclonal antibody directed at C5 and thus prevents the formation of the terminal complement complex. Eculizumab is successfully used in atypical haemolytic uraemic syndrome (aHUS) and paroxysomal nocturnal haemoglobinuria (PNH) but it is not standardly used in $\mathrm{LN}$. The aim of this project was to determine whether there is any role for eculizumab as adjunctive therapy in $L N$.

Methods: Using a predefined search strategy on Ovid MEDLINE and EMBASE the literature was reviewed systematically to identify studies in which eculizumab had been used to treat patients with SLE. All patients were included that were treated with complement inhibitors. Favourable outcome in this study was defined as resolution of symptoms that led to treatment, discharge from hospital or recovery of renal function. Patients were excluded if there was no outcome data or if complement inhibition was unrelated to their SLE.

Results: From 192 abstracts screened, 14 articles were identified, involving 30 patients. All SLE patients administered eculizumab were treated for thrombotic microangiopathy (TMA) secondary to LN diagnosed either histologically (66\%) or as part of a diagnosis of aHUS (73\%). 93\% of patients had a favourable outcome in response to eculizumab treatment, of which $46 \%$ had a favourable outcome and successfully stopped treatment without relapse in symptoms during a median follow up of 7 months. Three patients (10\%) reported adverse outcomes related to eculizumab therapy.

(Continued on next page)
\end{abstract}

\footnotetext{
* Correspondence: rdwright@liverpool.ac.uk

'Department of Women's and Children's Health, Institute of Life Course and

Medical Sciences, University of Liverpool, member of Liverpool Health

Partners, Eaton Road, Liverpool L12 2AP, UK

Full list of author information is available at the end of the article
}

C C The Author(s). 2020 Open Access This article is licensed under a Creative Commons Attribution 4.0 International License, which permits use, sharing, adaptation, distribution and reproduction in any medium or format, as long as you give appropriate credit to the original author(s) and the source, provide a link to the Creative Commons licence, and indicate if changes were made. The images or other third party material in this article are included in the article's Creative Commons licence, unless indicated otherwise in a credit line to the material. If material is not included in the article's Creative Commons licence and your intended use is not permitted by statutory regulation or exceeds the permitted use, you will need to obtain permission directly from the copyright holder. To view a copy of this licence, visit http://creativecommons.org/licenses/by/4.0/ The Creative Commons Public Domain Dedication waiver (http://creativecommons.org/publicdomain/zero/1.0/) applies to the data made available in this article, unless otherwise stated in a credit line to the data. 


\begin{abstract}
(Continued from previous page)
Conclusions: Scientific evidence supports the involvement of complement in the pathogenesis of LN however the role of complement inhibition in clinical practice is limited to those with TMA features. This systematic review showed that in cases of LN complicated with TMA, eculizumab seems to be a very efficacious therapy. Further evidence is required to determine whether patients with refractory $L N$ may benefit from adjunctive complement inhibition.
\end{abstract}

Keywords: Lupus nephritis, Eculizumab, Complement, Thrombotic microangiopathy, Systematic review

\section{Background}

Systemic lupus erythematosus (SLE) is a severe, systemic autoimmune condition that is characterised by inflammation and organ damage. SLE patients experience a loss of tolerance towards self-antigens due to autoreactive $B$ cells that produce autoantibodies, particularly against double-stranded DNA (dsDNA) and nuclear antigens. These autoantibodies correlate closely with disease activity and are used for diagnostic and prognostic evaluation [1]. Approximately 9.7/10,000 people in the UK suffer from SLE and people of Black Caribbean ethnicity have a higher incidence and prevalence of the disease; they are also more likely to have worsened disease manifestations compared to people of White ethnicity [2]. Lupus nephritis (LN) is one of the most common and damaging manifestations of SLE that affects approximately $40 \%$ of patients and leads to end-stage renal failure within 5 years in approximately 10\% [3]. Although evidenced-based therapeutic strategies are in place for LN (for instance use of disease modifying anti-rheumatic drugs (DMARDs) such as cyclophosphamide and mycophenolate mofetil [4]) and there are international recommendations for therapy (including the Single Hub and Access point for paediatric Rheumatology in Europe (SHARE) initiative recommendations [5]), many patients do not respond to therapy and the outcomes for LN are still poor (approximately 44\% of patients will enter remission [6]).

LN occurs in response to pathogenic autoantibodies in SLE binding IgG to form immune complexes which deposit in the kidney and result in damage, in part through activation of the complement cascade and innate immune system [7-10]. Thrombotic microangiopathy (TMA) is a form of endothelial injury that can occur in the kidneys of $1-4 \%$ of LN patients [11] where it appears to correlate with the most severe clinical manifestations and is associated with a high mortality [12].

The complement system is part of the innate immune response involved in recognition and opsonisation of invading pathogens, it also plays important roles in maintaining homeostasis through clearance of apoptotic debris and immune complexes [13]. Complement plays an important role in $\mathrm{LN}$ - deficiencies in components of the complement system (C1q, C2 and $\mathrm{C} 4)$ are prevalent in LN patients [14], particularly those that have a disease onset in childhood [15]. There is evidence to suggest that the alternative complement system can be pathogenically activated in SLE by the increased presence of immune complexes leading to overactivation of the terminal complement complex (C5b-9) and tissue damage [13, 16-18]. The kidney is particularly sensitive to immune complex deposition and thus complementmediated damage $[18,19]$. This is demonstrated through the increased levels of C5a and C5b-9 in the plasma of patients with active lupus nephritis [18].

Eculizumab is a recombinant fully humanised IgG2/ IgG4 monoclonal antibody that binds $\mathrm{C} 5$ and thus prevents the formation of the terminal complement complex [20]. It is currently used successfully in the treatment of atypical haemolytic uraemic syndrome (aHUS) and paroxysomal nocturnal hemoglobinuria $(\mathrm{PNH})[21,22]$. Eculizumab use is currently being explored in the treatment of other types of glomerulonephritis in which complement dysregulation is implicated including IgA nephropathy [23] and Shiga toxin-producing Escherichia coli (STEC-HUS) [24]. In view of its mode of action, eculizumab has also been considered for use in LN.

The aim of this project was to determine the role of eculizumab as adjunctive therapy in patients with LN. The objectives were to perform a systematic literature review using the PICOS framework - (P)articipants - all ages, sexes and ethnicities included, (I) ntervention those who received complement inhibition therapy for their SLE, $(C)$ omparison - before and after complement inhibition therapy, (O)utcome - any measurable outcome and (S)tudy design - any study design.

\section{Method}

\section{Search strategy}

We performed a systematic review of the literature, developed a priori, to identify case reports, clinical reports or clinical studies involving complement inhibiting therapies in patients with SLE. Keywords were identified and search terms used were: "LUPUS ERYTHEMATOSUS, SYSTEMIC" OR "systemic lupus erythematosus" (title, abstract) OR "lupus" (title, abstract) AND exp. "COMPLEMENT INACTIVATING AGENTS" OR "complement inhibitor" 
(title) OR "complement inhibition" (title) OR "eculizumab" (title) OR "soliris" (title) OR "avacopan" (title).

This search strategy was applied to the search engines Ovid MEDLINE and EMBASE from 2000 to present, this was intended to capture all patients who were treated with complement inhibition (first complement inhibition therapy, eculizumab, was approved for treatment of PNH in 2007). Results were filtered based on the availability of full text English language and all ages, sexes and ethnicities of patients were included. The search was conducted by FB on 17th May 2019.

\section{Patient population}

Patients were identified using the PICOS process (P)articipants - all ages, sexes and ethnicities included, (I)ntervention - those who received complement inhibition therapy for their SLE, (C)omparison - before and after complement inhibition therapy, (O)utcome - any measurable outcome and (S)tudy design - any study design. The inclusion criteria for this study was all patients with SLE who had received complement inhibition therapy as treatment for their SLE with any age, sex or ethnicity. Patients were excluded if eculizumab was administered for a condition unrelated to their SLE or if there was no data available on the outcome. Outcome was defined as response to eculizumab therapy favourable outcome was defined as resolution of the symptoms that led to treatment, discharge from hospital or recovery of renal function. Unfavourable outcome was defined as continuation of symptoms that lead to treatment or death. Adverse effects were defined as any negative effect that occurred during eculizumab therapy unrelated to their primary SLE. Where no data were available for a particular outcome characteristic, this was excluded from the analyses.

\section{Data collection}

Studies were identified through the above criteria (performed by FB, checked by RW) and were analysed independently by two reviewers (RW and LO) by abstract screening. Each manuscript was evaluated using full text to establish the indication for eculizumab treatment, previous medications, demographics, protocol used and outcome. Discrepancies on clinical features were resolved by consensus (RW and LO). Data were summarised qualitatively due to a lack of quantitative data associated with the studies.

\section{Ethics}

In accordance with NHS Health research authority guidance, ethical approval was not required as this systematic review included extracting data from studies that present anonymised individual patient data.

\section{Results}

\section{Search results}

The search strategy identified 214 records using the Ovid MEDLINE and EMBASE search engines, following removal of duplicates, 192 abstracts were screened. Of these, 172 were declared not suitable ( 174 by RW, 172 by LO) and 20 full-text articles were accessed for detailed inspection. Six further articles were excluded at this stage (involving 7 patients): two involved the same patient cases duplicated between published journals and published conference abstracts, one study reported a patient with SLE who was excluded before the end of the study, one study looked at four patients, two of whom had lupus but the results were not stratified by diagnosis and so were uninterpretable, one study reported a patient with SLE who was given eculizumab to treat an episode of aHUS caused by Dengue fever [25], and one study reported a patient with inactive SLE who was given eculizumab to treat Degos disease [26]. Therefore, 14 papers were included in the systematic literature review [21, 27-39] (Fig. 1). Inter-rater agreement at the abstract and full article stages were determined by Cohen's kappa coefficient ( 0.94 and 1 respectively).

\section{Patient population}

Characteristics of the 14 studies included in the systematic review are summarised in Table 1 . Altogether 30 patients were included in the analyses and of these $80 \%$ $(24 / 30)$ were female with a median age of 30 [range 459]. Two $(7 \%, 2 / 28)$ of the SLE patients included in this study were children $(<18$ years) and of those with data available there were six patients that were newly diagnosed $(30 \%, 6 / 20)$. Of the patients with a known diagnosis of SLE, three had childhood onset SLE but were adults when eculizumab was administered $(21 \%, 3 / 14)$. The studies predominantly came from the United States $(70 \%, 21 / 30)$ with the rest from Europe $(27 \%, 8 / 30)$ and Brazil (3\%, 1/30).

\section{Indications}

The indication for eculizumab therapy in all cases was a diagnosis of TMA secondary to active LN. The secondary TMA was determined either histologically (66\%, 19/ 30 ) or by diagnosis of aHUS (a combination of microangiopathic haemolytic anaemia, thrombocytopenia and renal impairment $(73 \%, 22 / 30))$.

Medications attempted prior to eculizumab treatment were varied. However, all patients (where data were available $(73 \%, 22 / 30))$ had previously been treated with corticosteroids and the majority of these had received plasma exchange therapy $(77 \%, 17 / 22)$. The most common other treatments used among these patients were: cyclophosphamide $(55 \%, 12 / 22)$, mycophenolate mofetil $(45 \%, 10 / 22)$ and rituximab $(41 \%, 9 / 22)$. 


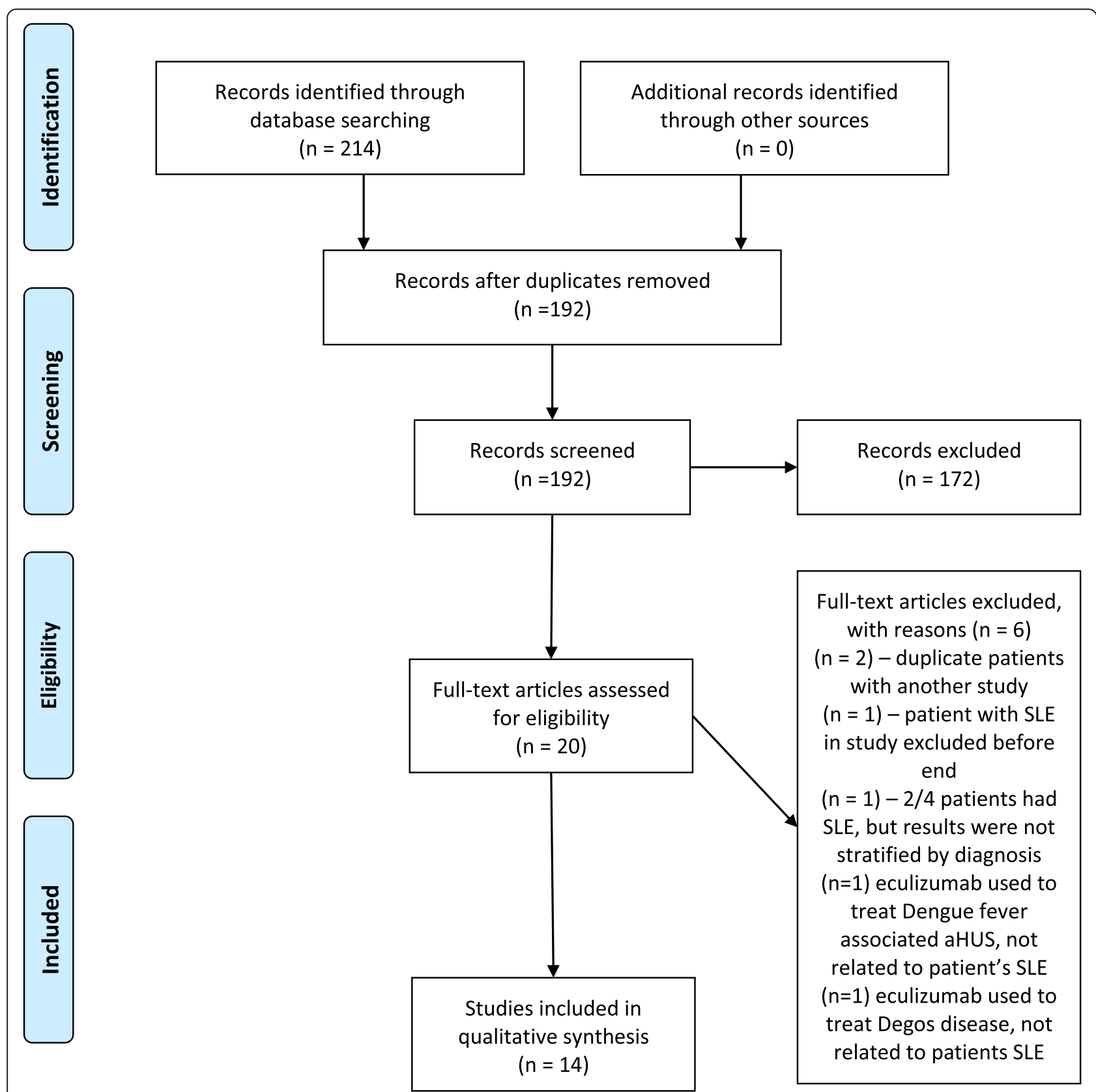

Fig. 1 PRISMA 2009 Flow Diagram for search strategy

\section{Eculizumab protocols}

The majority of patients (including one paediatric patient aged 16 years old) followed the Food and Drug Administration (FDA)-approved dosing schedule [40], namely four weekly doses of $900 \mathrm{mg}$ IV followed by $1200 \mathrm{mg}$ IV doses every other week (82\%, 23/28). However, a proportion of these patients never reached the $1200 \mathrm{mg}$ doses due to rapid remission of disease symptoms $(22 \%, 5 / 23)$. The remaining five patients for whom data were available followed slightly different protocols. One patient received a single $900 \mathrm{mg}$ IV dose followed by two weekly $1200 \mathrm{mg}$ doses, one patient received 1200 mg weekly for 4 weeks followed by $1200 \mathrm{mg}$ every 2 weeks. Another patient received $1200 \mathrm{mg}$ every 2 weeks, one patient had a single $1200 \mathrm{mg}$ dose followed by four weekly $1200 \mathrm{mg}$ doses and a final paediatric patient (4 years old) received $300 \mathrm{mg}$ every 2 weeks.

\section{Outcomes}

The majority of patients had favourable outcomes of eculizumab therapy $(93 \%, 28 / 30)$. Favourable outcome was defined as resolution of the symptoms that led to 
Table 1 Demographic and clinical data of LN patients treated with eculizumab. aHUS- atypical haemolytic uraemic syndrome, CYCcyclophosphamide, F- female, Gl- gastrointestinal, G- global, HCQ- hydroxychloroquine, IV- intravenous, LN- lupus nephritis, M- male, MMF- mycophenolate mofetil, PEX- plasma exchange, TMA- thrombotic microangiopathy, YO- year old

\begin{tabular}{|c|c|c|c|c|c|c|}
\hline Reference & $\begin{array}{l}\text { Number of } \\
\text { opstitents } \\
\text { sex, racese, }\end{array}$ & Indication & Previous medications & Protocol & Outcome & follow-up \\
\hline \multirow{7}{*}{$\begin{array}{l}\text { Kello, , et al, } \\
2018^{\circ:}\end{array}$} & 1 (27Yo F) & \multirow{7}{*}{ 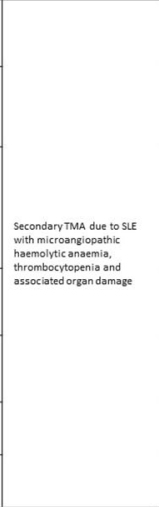 } & Steroids and CYC & 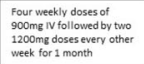 & $\begin{array}{l}\text { Disease-free at } \\
\text { followup }\end{array}$ & 26 months \\
\hline & $\begin{array}{l}\text { 1140rof } \\
\text { Hisponicl }\end{array}$ & & $\begin{array}{l}\text { Sterolids and } \\
\text { onticosuluants }\end{array}$ & 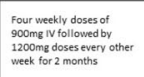 & 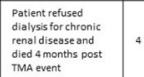 & 4 months \\
\hline & $\begin{array}{l}1 \text { (132YOF } \\
\text { African- } \\
\text { American) }\end{array}$ & & $\begin{array}{l}\text { Sterodids, } \\
\text { anticoogulants and } \\
\text { PEX }\end{array}$ & $\begin{array}{l}\text { Four weekly doses of } \\
\text { 9oomg iv followed by } \\
1220 \text { mg dosesevery other } \\
\text { week for } 8 \text { months }\end{array}$ & 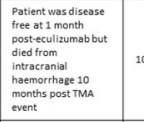 & 10 months \\
\hline & $\begin{array}{l}1 \text { (27rom } \\
\text { coucesian) }\end{array}$ & & $\begin{array}{l}\text { Steroids, PEX and } \\
\text { MMF }\end{array}$ & 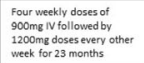 & $\begin{array}{l}\text { Disease-free at } \\
\text { followup }\end{array}$ & 36 months \\
\hline & 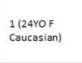 & & $\begin{array}{l}\text { Sterorids, PEX, } \\
\text { anticoegulants and } \\
\text { CrC }\end{array}$ & 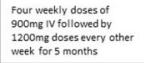 & $\begin{array}{l}\text { Disease-free at } 4 \\
\text { months post- } \\
\text { eculizumab }\end{array}$ & 12 months \\
\hline & $\begin{array}{l}1(21 \mathrm{yoM} M \\
\text { Asian) }\end{array}$ & & $\begin{array}{l}\text { Steroids, PEX and } \\
\text { Rituximab }\end{array}$ & $\begin{array}{l}\text { Four weekly doses of } \\
\text { 9oomg IV }\end{array}$ & $\begin{array}{l}\text { Disease-fiee at 1 } \\
\text { month post } \\
\text { ecullizumbib }\end{array}$ & 12 months \\
\hline & $\begin{array}{l}1 \text { (21YOMM } \\
\text { Africar- } \\
\text { Anerican) }\end{array}$ & & Steroids, PEX and CYC & $\begin{array}{l}\text { four weekly doses of } \\
900 \mathrm{mg} \text { IV }\end{array}$ & $\begin{array}{l}\text { Disessefree at } 1 \\
\text { month post- } \\
\text { eculizumab }\end{array}$ & 12 months \\
\hline $\begin{array}{l}\text { Park, Mand } \\
\text { Weitz IC. } \\
2017^{21}\end{array}$ & $\begin{array}{l}\text { 8(Median } \\
\text { oge } 34 \text { [21- } \\
\text { 59] and } 7 / 8 \\
\text { F) }\end{array}$ & 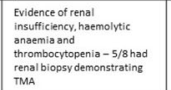 & No data aval lable & 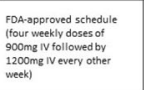 & 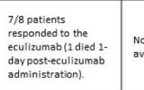 & $\begin{array}{l}\text { No doto } \\
\text { availibble }\end{array}$ \\
\hline 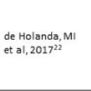 & 1 (18Yo F) & $\begin{array}{l}\text { Fever, abdominal opain, } \\
\text { dysuruia, skin rash and oligo- } \\
\text { anuria. Oilagnosed with SLE } \\
\text { with LN and TMA }\end{array}$ & 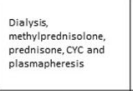 & 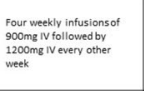 & $\begin{array}{l}\text { Patient recovered } \\
\text { ond was dischorged } \\
32 \text { days after first } \\
\text { eecuitumabobose } \\
\text { patient remains on } \\
\text { eculizumab }\end{array}$ & 3 vears \\
\hline $\begin{array}{l}\text { Geethakumari } \\
\text { Pe Ret al, 2017 }\end{array}$ & $\begin{array}{l}1 \text { 158ro M } \\
\text { Caucasien) }\end{array}$ & $\begin{array}{l}\text { Abdominal pain post- } \\
\text { transplant(pre-emptive } \\
\text { unneralted living donoro) - } \\
\text { briosssy confirmed TMA }\end{array}$ & 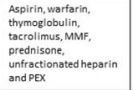 & 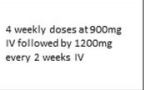 & $\begin{array}{l}\text { Stable renal function } \\
\text { - patient remains on } \\
\text { eculizumab }\end{array}$ & >20 months \\
\hline \multirow{4}{*}{$\begin{array}{l}\text { Covero, Tet ol, } \\
\text { 2017' }\end{array}$} & 1 (5190) & \multirow{4}{*}{$\begin{array}{l}\text { Secondary aHus with TMA } \\
\text { ond persistence of TMA } \\
\text { desepite traztment of cause } \\
\text { and plasmapharesis }\end{array}$} & 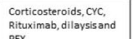 & $\begin{array}{l}\text { Four weekiy doseses of } \\
900 \mathrm{mg} \text { IV }\end{array}$ & $\begin{array}{l}\text { Petient remains on } \\
\text { dialyss sond }\end{array}$ & 0.9 months \\
\hline & $1(16 \% 0 F)$ & & & Four weekly doses of & ecullzumab & \\
\hline & \multirow{2}{*}{ 1(5270 F) } & & $\begin{array}{l}\text { Corticosteroids and } \\
\text { MMF }\end{array}$ & 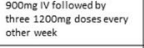 & $\begin{array}{l}\text { Patient remains on } \\
\text { dialysis }\end{array}$ & 4.2 months \\
\hline & & & $\begin{array}{l}\text { Corticosteroids, } \\
\text { cyclosporine and PEX }\end{array}$ & $\begin{array}{l}\text { Two weckly doses of } \\
\text { 9000m IV }\end{array}$ & $\begin{array}{l}\text { Reduction in serum } \\
\text { creatinine }\end{array}$ & 4 months \\
\hline $\begin{array}{l}\text { Sarkissian, S. } \\
2016^{24}\end{array}$ & $1(25 \% \mathrm{~F})$ & $\begin{array}{l}\text { Renal biopsyshowed classiv } \\
\text { LN with TMA }\end{array}$ & $\begin{array}{l}\text { Bloodtransfusions, } \\
\text { steroids, Rituximab, } \\
\text { CrC and } \\
\text { plasmapheresis }\end{array}$ & No data availsble & $\begin{array}{l}\text { Patient beccame } \\
\text { trtansfusion } \\
\text { independent with } \\
\text { improved creatinine }\end{array}$ & 14 doys \\
\hline $\begin{array}{l}\text { Roufi,A G et ol. } \\
20166^{23}\end{array}$ & $\begin{array}{l}1(2550 F \\
\text { Vietrnmeses) }\end{array}$ & 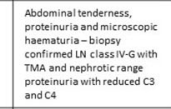 & 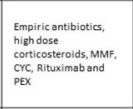 & $\begin{array}{l}4 \text { weekly doseses atsooomg } \\
\text { IV followededy } 12000 \mathrm{mg} \\
\text { everv } 2 \text { weeks iv }\end{array}$ & $\begin{array}{l}\text { Significant renal } \\
\text { recovery and } \\
\text { repress inn of } \\
\text { haemmlyticactivity- } \\
\text { patient remains on } \\
\text { eeculizumab }\end{array}$ & 32 weeks \\
\hline \multirow[b]{2}{*}{$\begin{array}{l}\text { Bermea, RS et } \\
\text { al, 2016 }\end{array}$} & $\begin{array}{l}1 \text { (300vo F } \\
\text { African- } \\
\text { American) }\end{array}$ & 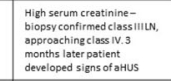 & $\begin{array}{l}\text { IV } \\
\text { methylpreddisolione, } \\
\text { Cr, Prex, } \\
\text { Immunoglobulin }\end{array}$ & $\begin{array}{l}\text { 900mg I I f followed by } \\
1200 \mathrm{mg} \text { every other week } \\
\text { for } 2 \text { months }\end{array}$ & $\begin{array}{l}\text { Immediate } \\
\text { Improvement of } \\
\text { renal svmpotoms }\end{array}$ & 5 months \\
\hline & $\begin{array}{l}1 \text { (21Yom } \\
\text { Hispanic) }\end{array}$ & $\begin{array}{l}\text { Thrombocrtopenia and } \\
\text { wor'sening renal tunction } \\
\text { vith presence of schistortes } \\
\text { confirmed aHus }\end{array}$ & $\begin{array}{l}\text { MMF, HCQ, steroids, } \\
\text { PEX, Rituximab, CCYC }\end{array}$ & $\begin{array}{l}900 \mathrm{mg} \text { IV weekly for } 2 \\
\text { weeks }\end{array}$ & 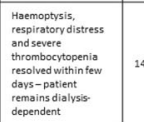 & 14 months \\
\hline $\begin{array}{l}\text { Pickering,Mc } \\
\text { etat, 2015? }\end{array}$ & $\begin{array}{l}1 \text { (19vo F } \\
\text { caucasian) }\end{array}$ & 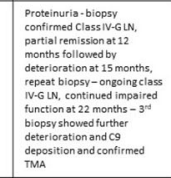 & $\begin{array}{l}\text { Prednisolone, HCa iv } \\
\text { Crc, Bituximab, MMF } \\
\text { and tacrolimus }\end{array}$ & 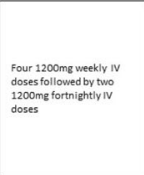 & $\begin{array}{l}\text { Rapid and sustained } \\
\text { improved renal } \\
\text { function }\end{array}$ & 40 months \\
\hline 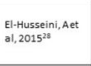 & $\begin{array}{l}1 \text { (2avo of } \\
\text { African- } \\
\text { American) }\end{array}$ & $\begin{array}{l}\text { Anaemia, thrombocitopenis } \\
\text { and acute kidney injury- } \\
\text { biopsy conflirmed TMA }\end{array}$ & $\begin{array}{l}\text { Prednisone, MMF, } \\
\text { Crc, plasmapheresis } \\
\text { andiv } \\
\text { methyiprednisolone }\end{array}$ & $\begin{array}{l}1200 \text { me everv } 2 \text { weeks IV } \\
\text { for } 6 \text { months }\end{array}$ & $\begin{array}{l}\text { Renal symptoms } \\
\text { subsided within 2 } \\
\text { weeks }\end{array}$ & 12 months \\
\hline $\begin{array}{l}\text { Coppo Ret al, } \\
20155^{\circledR}\end{array}$ & $\begin{array}{l}\text { larof } \\
\text { Moroccan) }\end{array}$ & 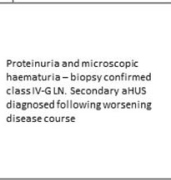 & 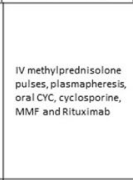 & 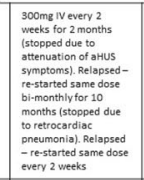 & $\begin{array}{l}\text { Strable renal function- } \\
\text { patient remained on } \\
\text { ecul zummab }\end{array}$ & ${ }_{\text {months }}^{22}$ \\
\hline $\begin{array}{l}\text { Kronbichler, } A \\
\text { et al, 2014 }\end{array}$ & 1 (30YO F) & 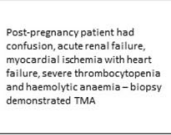 & 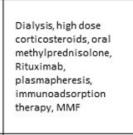 & 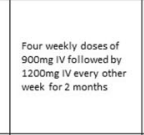 & 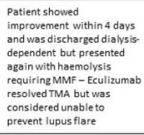 & : \\
\hline $\begin{array}{l}\text { Pierer, Met al, } \\
2013^{11}\end{array}$ & 1(F) & 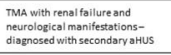 & PEX and dialysis & $\begin{array}{l}\text { No dosing dato } \\
\text { avaizablo-eculizumab } \\
\text { given for } 12 \text { months }\end{array}$ & $\begin{array}{l}\text { Pattient improved rapidly- } \\
\text { renal function remains } \\
\text { stable }\end{array}$ & $\begin{array}{l}12 \\
\text { months }\end{array}$ \\
\hline $\begin{array}{l}\text { Hadaya, Ket al, } \\
\text { 20112, }\end{array}$ & 1 (27\%OF) & 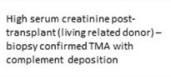 & 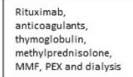 & 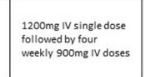 & $\begin{array}{l}\text { Completet resolution of TMA } \\
\text { with reduced serum } \\
\text { creatinire and normal ised } \\
c 3 / C_{4} 4 \text { levels }\end{array}$ & ${ }_{\text {months }}^{6}$ \\
\hline
\end{tabular}


treatment $(86 \%, 24 / 28)$, discharge from hospital $(82 \%$, $23 / 28)$, or recovery of renal function $(75 \%, 21 / 28)$ (two of these patients died in a manner reported to be unrelated to eculizumab therapy or TMA, one from an intracranial haemorrhage and one patient declined dialysis). Recovery was rapid in patients who responded where data was available (median 2.5 weeks [range $0.75-16$ weeks]). Of the two patients who did not respond, one died within 1 day of eculizumab administration, but the reason was not provided. The other patient was followed up only until his final eculizumab dose (4 weeks) and remained dialysis dependent (Fig. 2).

Of the 28 patients who responded to treatment, $46 \%$ $(13 / 28)$ successfully stopped eculizumab treatment, $36 \%$ $(10 / 28)$ had no data available and 18\% (5/28) are still receiving eculizumab treatment (1 patient stopped treatment twice and relapsed requiring repeat doses). There was a short median follow up time of 7 months [range 0.45-40] (Fig. 2).

The majority of patients $(90 \%$ - $27 / 30)$ reported no adverse events in response to eculizumab therapy. One study recorded a patient exhibiting acute pancreatitis, diarrhoea and pneumonia that required 3 stays in hospital over the 6-month course of eculizumab treatment however it was not stated whether this was believed to be directly related to the eculizumab therapy. Another study reported an incidence of nausea without vomiting in a patient on eculizumab and another of nausea with vomiting, however both resolved within $24 \mathrm{~h}$ and did not recur during therapy.

\section{Discussion}

The role of the complement cascade in $\mathrm{LN}$ is well evidenced; deficiencies in components of the complement cascade can lead to monogenic lupus (C1q, C2 and C4) [14] and low levels of C3 and C4 are assessed in the clinic to monitor disease activity. Further glomerular deposition of complement is almost universally seen in histological analysis. A study of 222 Chinese patients with active LN demonstrated that the alternative complement pathway may be important in the pathogenesis of $\mathrm{LN}$ and that factor $\mathrm{Bb}$ may be a biomarker for measuring disease activity [18]. Another study of $30 \mathrm{LN}$ patients showed increased deposition of C9 in glomerular biopsy tissue in patients with worse disease compared to those with a more mild phenotype suggesting that the terminal complement complex (C5b-9) may be a suitable biomarker for LN disease activity [41].

Despite the above evidence that complement is important in the pathogenesis of $\mathrm{LN}$, current therapies for LN are broad spectrum immunosuppressants and complement inhibitors are not routinely used. Eculizumab is both effective and safe in the treatment of aHUS [42]. It is well recognised that LN patients can develop secondary aHUS and TMA. However, despite the efficacy of eculizumab in aHUS patients this is not used as first line treatment in these patients. This review summarises case reports and studies that suggest that the use of eculizumab in LN complicated by TMA can be beneficial to patients with excellent response rates described after a short course of treatment. Further only 3 patients reported adverse events during eculizumab therapy

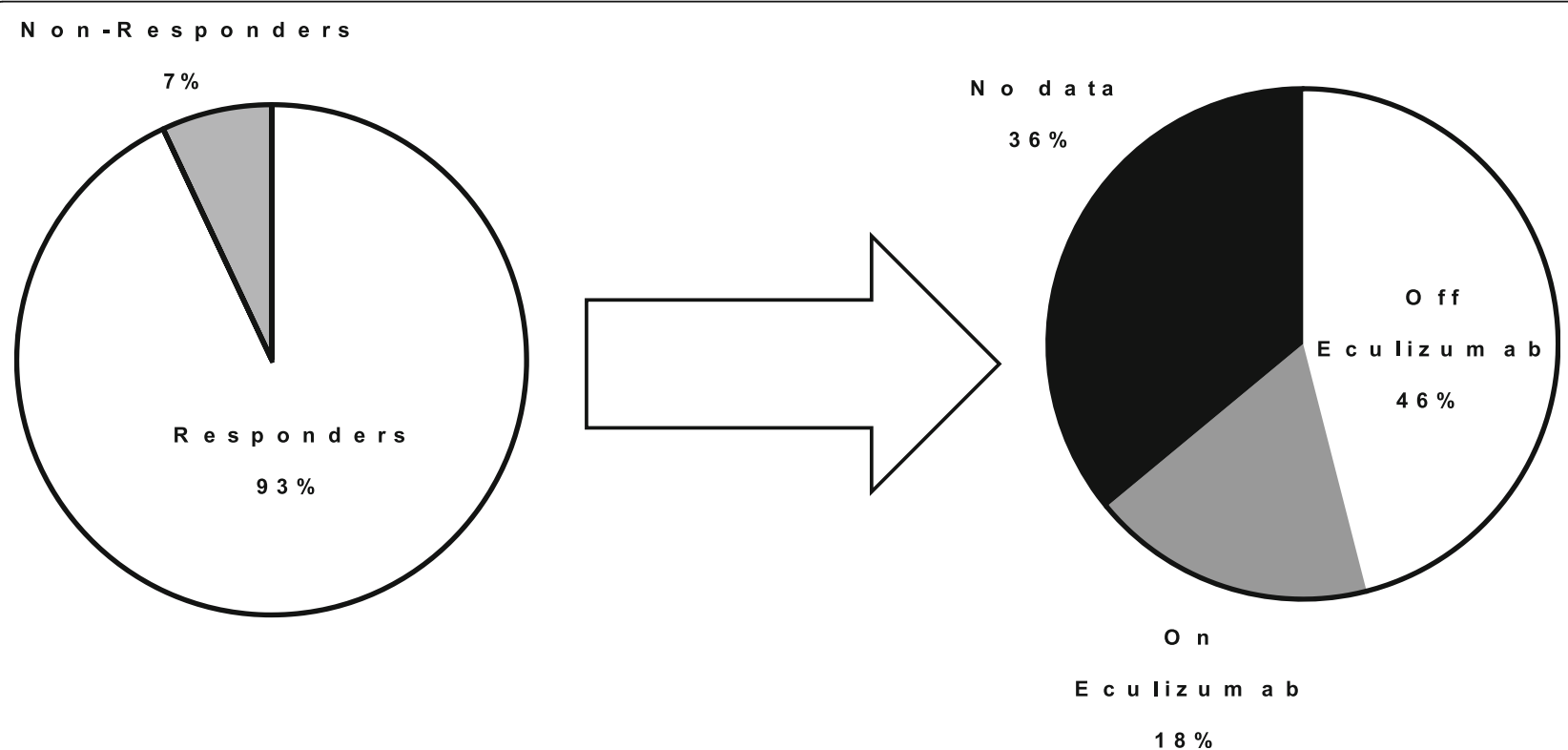

Fig. 2 Patient response to Eculizumab therapy. 93\% of patients to whom eculizumab was administered responded favourably. Of these $46 \%$ were withdrawn from treatment, 18\% remained on treatment at follow up and $36 \%$ had no data available 
suggesting that the safety profile of the drug is good. Eculizumab may have a role in refractory LN but this systemic literature review did not reveal any reported cases and the cost of this drug may be prohibitive [43].

TMA is seen in a subset of LN patients (1-4\%) [11] and a small study of 11 patients demonstrated that $60 \%$ of these had complement regulatory protein mutations previously associated with aHUS [44]. No other studies have analysed the association between SLE patients with complement mutations and the prevalence of TMA however this is an area that requires more investigation.

Further mechanistic work is required to determine whether it is possible to identify patients with $\mathrm{LN}$ who may benefit from these medications as it may be possible to stratify patients. Correlating clinical phenotype with markers of complement activation would help to determine which patients should receive complement inhibition, at what point in disease course, and when is it safe to stop. The use of adjunctive treatment such as Eculizumab to current management protocols may improve the suboptimal renal outcomes in patients with LN.

One limitation of this study is that data related to the serological or non-renal parameters of disease is only available for four of the 30 patients included. This data may provide increased evidence on the risks or benefits of using eculizumab for LN-associated TMA in SLE patients.

\section{Conclusion}

This systematic review has demonstrated that clinically, eculizumab is a treatment option in secondary aHUS or TMA in patients with LN. Patients with refractory LN may benefit from adjunctive treatment with complement inhibition but further studies are required to define its role in this disease.

\section{Abbreviations}

aHUS: Atypical haemolytic uraemic syndrome; DMARDs: Disease modifying anti-rheumatic drugs; dsDNA: Double stranded DNA; LN: Lupus nephritis; PNH: Paroxysomal nocturnal haemoglobinuria; SHARE: Single Hub and Access point for paediatric Rheumatology in Europe; SLE: Systemic lupus erythematosus; STEC-HUS: Shiga toxin-producing Escherichia coli - haemolytic uraemic syndrome; TMA: Thrombotic microangiopathy

\section{Acknowledgements \\ The authors would like to thank colleagues within the UK's 'Experimenta Arthritis Treatment Centre for Children' (www.EATC4Children.co.uk), the National Institute of Health Research Alder Hey Clinical Research Facility for Experimental Medicine, and in particular the Lupus Research Group, supported by LUPUS UK, for their support and comments on this work.}

\section{Authors' contributions}

Conceptualization, R.D.W. and L.O.; Methodology, R.D.W. and L.O.; Investigation, R.D.W, F.B., and L.O..; Writing - Original Draft, R.D.W.; Writing Review \& Editing, R.D.W, F.B., M.W.B., and L.O.; Funding Acquisition, R.D.W., and L.O. All authors have read and approved the manuscript.

\section{Funding}

This project received funding from Alder Hey Children's Kidney Fund, the UK's Experimental Arthritis Treatment Centre for Children (supported by
Versus Arthritis, Alder Hey Children's NHS Foundation Trust, the Alder Hey Charity, and the University of Liverpool), LUPUS UK, and the National Institute of Health Research (NIHR) Alder Hey Clinical Research Facility for Experimental Medicine.

Availability of data and materials

Not applicable.

Ethics approval and consent to participate

Not applicable.

\section{Consent for publication}

Not applicable.

\section{Competing interests}

The authors declare that they have no competing interest.

\section{Author details}

${ }^{1}$ Department of Women's and Children's Health, Institute of Life Course and Medical Sciences, University of Liverpool, member of Liverpool Health Partners, Eaton Road, Liverpool L12 2AP, UK. '2ibrary and Knowledge Service, Alder Hey Children's NHS Foundation Trust, member of Liverpool Health Partners, Liverpool, UK. ${ }^{3}$ Department of Paediatric Rheumatology, Alder Hey Children's NHS Foundation Trust, member of Liverpool Health Partners, Liverpool, UK. ${ }^{4}$ Department of Paediatric Nephrology, Alder Hey Children's NHS Foundation Trust, member of Liverpool Health Partners, Liverpool, UK.

Received: 26 July 2019 Accepted: 8 June 2020

Published online: 30 June 2020

\section{References}

1. Riboldi P, Gerosa M, Moroni G, et al. Anti-DNA antibodies: a diagnostic and prognostic tool for systemic lupus erythematosus? Autoimmunity. 2005;38: 39-45. https://doi.org/10.1080/08916930400022616.

2. Korbet SM, Schwartz MM, Evans J, et al. Severe lupus nephritis: racial differences in presentation and outcome. J Am Soc Nephrol. 2007:18:24454. https://doi.org/10.1681/ASN.2006090992.

3. Hoover PJ, Costenbader KH. Insights into the epidemiology and Management of Lupus Nephritis from the U.S. Rheumatologist's perspective. Kidney Int. 2016;90:487-92. https://doi.org/10.1016/j.kint.2016.03.042.

4. Bertsias GK, Tektonidou M, Amoura Z, et al. Joint European League Against Rheumatism and European Renal Association-European Dialysis and Transplant Association (EULAR/ERA-EDTA) recommendations for the management of adult and paediatric lupus nephritis. Ann Rheum Dis 2012; 71: 1771-1782. 2012/08/02. DOI: https://doi.org/10.1136/annrheumdis-2012201940

5. Groot N, de Graeff N, Marks SD, et al. European evidence-based recommendations for the diagnosis and treatment of childhood-onset lupus nephritis: the SHARE initiative. Ann Rheum Dis 2017; 76: 1965-1973. 2017/09/08. DOI: https://doi.org/10.1136/annrheumdis-2017-211898.

6. Zampeli E, Klinman DM, Gershwin ME, et al. A comprehensive evaluation for the treatment of lupus nephritis. J Autoimmun 2017; 78: 1-10. 2017/01/14 DOl: https://doi.org/10.1016/j.jaut.2016.12.011.

7. Davidson A. What is damaging the kidney in lupus nephritis? Nat Rev Rheumatol 2016; 12: 143-153. 2015/11/19. DOl: https://doi.org/10.1038/ nrrheum.2015.159.

8. Wang $H, X u$ J, Zhang $X$, et al. Tubular basement membrane immune complex deposition is associated with activity and progression of lupus nephritis: a large multicenter Chinese study. Lupus 2018; 27: 545-555. 2017/ 09/29. DOl: https://doi.org/10.1177/0961203317732407.

9. Sugisaki T and Takase S. Composition of immune deposits present in glomeruli of NZB/W F1 mice. Clin Immunol Immunopathol 1991; 61: 296308. 1991/12/01. DOl: https://doi.org/10.1016/s0090-1229(05)80002-9.

10. Weening JJ, D'Agati VD, Schwartz MM, et al. The classification of glomerulonephritis in systemic lupus erythematosus revisited. J Am Soc Nephrol. 2004;15:241-50.

11. Lansigan F, Isufi I and Tagoe CE. Microangiopathic haemolytic anaemia resembling thrombotic thrombocytopenic purpura in systemic lupus erythematosus: the role of ADAMTS13. Rheumatology (Oxford) 2011; 50: 824-829. 2010/12/15. DOI: https://doi.org/10.1093/rheumatology/keq395. 
12. Song $D$, Wu LH, Wang FM, et al. The spectrum of renal thrombotic microangiopathy in lupus nephritis. Arthritis Res Ther 2013; 15: R12. 2013/ 01/17. DOI: https://doi.org/10.1186/ar4142.

13. Ricklin D, Reis ES and Lambris JD. Complement in disease: a defence system turning offensive. Nat Rev Nephrol 2016; 12: 383-401. 2016/05/24. DOI: https://doi.org/10.1038/nrneph.2016.70.

14. Costa-Reis $P$ and Sullivan KE. Monogenic lupus: it's all new! Curr Opin Immunol 2017; 49: 87-95. 2017/11/04. DOl: https://doi.org/10.1016/j.coi. 2017.10.008.

15. Pereira KM, Faria AG, Liphaus BL, et al. Low C4, C4A and C4B gene copy numbers are stronger risk factors for juvenile-onset than for adult-onset systemic lupus erythematosus. Rheumatology (Oxford) 2016; 55: 869-873. 2016/01/24. DOl: https://doi.org/10.1093/rheumatology/kev436.

16. Leffler J, Bengtsson AA and Blom AM. The complement system in systemic lupus erythematosus: an update 2014. DOI: https://doi.org/10.1136/ annrheumdis-2014-205287

17. Chen M, Daha MR and Kallenberg CG. The complement system in systemic autoimmune disease. J Autoimmun 2010; 34: J276-J286. 2009/12/17. DOI: https://doi.org/10.1016/j.jaut.2009.11.014

18. Song D, Guo WY, Wang FM, et al. Complement Alternative Pathways Activation in Patients With Lupus Nephritis. Am J Med Sci 2017; 353: $247-$ 257. 2017/03/07. DOl: https://doi.org/10.1016/j.amjms.2017.01.005.

19. Mizuno $M$, Suzuki $Y$ and Ito $Y$. Complement regulation and kidney diseases: recent knowledge of the double-edged roles of complement activation in nephrology. Clin Exp Nephrol 2018; 22: 3-14. 2017/03/28. DOI: https://doi. org/10.1007/s10157-017-1405-x

20. Kaplan M. Eculizumab (Alexion). Curr Opin Investig Drugs 2002; 3: 1017 1023. 2002/08/21

21. Cavero T, Rabasco C, López A, et al. Eculizumab in secondary atypical haemolytic uraemic syndrome. Nephrol Dial Transplant. 2017:466-74.

22. Socie G, Caby-Tosi MP, Marantz JL, et al. Eculizumab in paroxysmal nocturnal haemoglobinuria and atypical haemolytic uraemic syndrome: 10year pharmacovigilance analysis. Br J Haematol 2019; 185: 297-310. 2019/ 02/16. DOl: https://doi.org/10.1111/bjh.15790.

23. Rizk DV, Maillard N, Julian BA, et al. The Emerging Role of Complement Proteins as a Target for Therapy of IgA Nephropathy. Front Immunol 2019; 10: 504. 2019/04/04. DOl: https://doi.org/10.3389/fimmu.2019.00504.

24. Walsh PR and Johnson S. Treatment and management of children with haemolytic uraemic syndrome. Arch Dis Child 2018; 103: 285-291. 2017/09/ 14. DOI: https://doi.org/10.1136/archdischild-2016-311377.

25. Khandelwal A, Wright JK, Pavenski K, et al. Risks of novel therapeutics: gonococcemia in an immune-suppressed patient receiving eculizumab. CMAJ 2017; 189: E1558-e1560. 2017/12/20. DOI: https://doi.org/10.1503/ cmaj.170508.

26. Dayanand S, Sclair S, Enrico S. An unusual case of gastrointestinal bleeding that resolved with eculizumab infusion. Am J Gastroenterol. 2014;109.

27. Kello N, Khoury LE, Marder G, et al. Secondary thrombotic microangiopathy in systemic lupus erythematosus and antiphospholipid syndrome, the role of complement and use of eculizumab: Case series and review of literature. Semin Arthritis Rheum 2018 2019/01/02. DOI: https://doi.org/10.1016/j. semarthrit.2018.11.005

28. Park M, Weitz I. Effective treatment of thrombotic microangiopathy associated with lupus nephritis with eculizumab: a series of 8 cases. Blood. 2017;130:1043

29. De Holanda MI, Porto LC, Wagner T, et al. Use of eculizumab in a systemic lupus erythemathosus patient presenting thrombotic microangiopathy and heterozygous deletion in CFHR1-CFHR3. A case report and systematic review. Clin Rheumatol 2017; 36: 2859-2867. 2017/09/15. DOl: https://doi. org/10.1007/s10067-017-3823-2.

30. Geethakumari PR, Mille P, Gulati R, et al. Complement inhibition with eculizumab for thrombotic microangiopathy rescues a living-donor kidney transplant in a patient with antiphospholipid antibody syndrome. Transfus Apher Sci 2017; 56: 400-403. 2017/04/22. DOI: https://doi.org/10.1016/j. transci.2017.02.007.

31. Sarkissian S. Successful treatment of acute renal failure in patient with active lupus nephritis and atypical hemolytic uremic syndrome. J Investig Med. 2016;64

32. Raufi AG, Scott S, Darwish O, et al. Atypical Hemolytic Uremic Syndrome Secondary to Lupus Nephritis, Responsive to Eculizumab. Hematol Rep 2016; 8: 6625. 2016/10/27. DOl: https://doi.org/10.4081/hr.2016.6625.
33. Bermea RS, Sharma N, Cohen K, et al. Use of Eculizumab in Atypical Hemolytic Uremic Syndrome, Complicating Systemic Lupus Erythematosus. J Clin Rheumatol 2016; 22: 320-323. 2016/08/25. DOl: https://doi.org/10. 1097/rhu.0000000000000423.

34. Pickering $M C$, Ismajli $M$, Condon MB, et al. Eculizumab as rescue therapy in severe resistant lupus nephritis. Rheumatology (Oxford). Engl. 2015:2286-8.

35. El-Husseini A, Hannan S, Awad A, et al. Thrombotic microangiopathy in systemic lupus erythematosus: efficacy of eculizumab. Am J Kidney Dis 2015; 65: 127-130. 2014/12/03. DOl: https://doi.org/10.1053/j.ajkd.2014.07. 031.

36. Coppo R, Peruzzi L, Amore A, et al. Dramatic effects of eculizumab in a child with diffuse proliferative lupus nephritis resistant to conventional therapy. Pediatr Nephrol 2015; 30: 167-172. 2014/09/01. DOl: https://doi.org/10.1007/ s00467-014-2944-y.

37. Kronbichler A, Frank R, Kirschfink $M$, et al. Efficacy of eculizumab in a patient with immunoadsorption-dependent catastrophic antiphospholipid syndrome: a case report. Medicine (Baltimore) 2014; 93: e143. 2014/12/05. DOl: https://doi.org/10.1097/md.0000000000000143.

38. Pierer M, Luttosch F, Baerwald C. Role of complement-inhibition in thrombotic microangiopathy (TMA) associated with systemic lupus erythematosus (SLE): a case report. Z Rheumatol. 2013;72.

39. Hadaya K, Ferrari-Lacraz S, Fumeaux D, et al. Eculizumab in acute recurrence of thrombotic microangiopathy after renal transplantation. Am J Transplant 2011; 11: 2523-2527. 2011/08/13. DOl: https://doi.org/10.1111/j.1600-6143. 2011.03696.x.

40. Kaplan BS, Ruebner RL, Spinale JM, et al. Current treatment of atypical hemolytic uremic syndrome. Intractable Rare Dis Res. 2014;3:34-45. https:// doi.org/10.5582/irdr.2014.01001

41. Wang S, Wu M, Chiriboga L, et al. Membrane attack complex (mac) deposition in lupus nephritis is associated with hypertension and poor clinical response to treatment. Semin Arthritis Rheum 2018; 48: 256-262. 2018/02/06. DOI: https://doi.org/10.1016/j.semarthrit.2018.01.004.

42. Menne J, Delmas $Y$, Fakhouri F, et al. Outcomes in patients with atypical hemolytic uremic syndrome treated with eculizumab in a long-term observational study. BMC Nephrol 2019; 20: 125. 2019/04/12. DOI: https:// doi.org/10.1186/s12882-019-1314-1

43. Eculizumab - with $£ 340,200$ price tag - gets go ahead from NICE. The Pharmaceutical Journal, 7 February 2015, Vol 294, No 7848, online. https:// doi.org/10.1211/PJ.2015.20067720.

44. Park MH, Caselman N, Ulmer S, et al. Complement-mediated thrombotic microangiopathy associated with lupus nephritis. Blood Adv. 2018, pp.20902094

\section{Publisher's Note}

Springer Nature remains neutral with regard to jurisdictional claims in published maps and institutional affiliations.
Ready to submit your research? Choose BMC and benefit from:

- fast, convenient online submission

- thorough peer review by experienced researchers in your field

- rapid publication on acceptance

- support for research data, including large and complex data types

- gold Open Access which fosters wider collaboration and increased citations

- maximum visibility for your research: over $100 \mathrm{M}$ website views per year

At $\mathrm{BMC}$, research is always in progress.

Learn more biomedcentral.com/submissions 